LA W RENCE LIVERMORE N A TIO NAL LABORATORY

\title{
Energy Return on Investment - Fuel Recycle
}

W. Halsey, A. J. Simon, M. Fratoni, C. Smith, P. Schwab, P. Murray

June 8, 2012 
This document was prepared as an account of work sponsored by an agency of the United States government. Neither the United States government nor Lawrence Livermore National Security, LLC, nor any of their employees makes any warranty, expressed or implied, or assumes any legal liability or responsibility for the accuracy, completeness, or usefulness of any information, apparatus, product, or process disclosed, or represents that its use would not infringe privately owned rights. Reference herein to any specific commercial product, process, or service by trade name, trademark, manufacturer, or otherwise does not necessarily constitute or imply its endorsement, recommendation, or favoring by the United States government or Lawrence Livermore National Security, LLC. The views and opinions of authors expressed herein do not necessarily state or reflect those of the United States government or Lawrence Livermore National Security, LLC, and shall not be used for advertising or product endorsement purposes.

This work performed under the auspices of the U.S. Department of Energy by Lawrence Livermore National Laboratory under Contract DE-AC52-07NA27344. 


\title{
Energy Return on Investment - Fuel Recycle
}

\author{
Patrick R. Schwab \\ U.S. Department of Energy \\ 19901 Germantown Road, Germantown, MD 20874 \\ Patrick.schwab@nuclear.energy.gov \\ William Halsey, A. J. Simon, Massimiliano Fratoni and Clara Smith \\ Lawrence Livermore National Laboratory \\ 7000 East Avenue, Livermore, CA 94550 \\ Halsey1@llnl.gov, Simon19@Ilnl.gov, Fratoni1@llnl.gov and Smith456@llnl.gov \\ Paul Murray \\ Areva Federal Services \\ Address \\ Paul.murray@areva.com
}

\section{INTRODUCTION}

This report provides a methodology and requisite data to assess the potential Energy Return On Investment (EROI) for nuclear fuel cycle alternatives, and applies that methodology to a limited set of used fuel recycle scenarios. This paper is based on a study by Lawrence Livermore National Laboratory [1] and a parallel evaluation by AREVA Federal Services LLC [2], both of which were sponsored by the DOE Fuel Cycle Technologies (FCT) Program. The focus of the LLNL effort was to develop a methodology that can be used by the FCT program for such analysis that is consistent with the broader energy modeling community, and the focus of the AREVA effort was to bring industrial experience and operational data into the analysis. This cooperative effort successfully combined expertise from the energy modeling community with expertise from the nuclear industry.

Energy Return on Investment is one of many figures of merit on which investment in a new energy facility or process may be judged. EROI is the ratio of the energy delivered by a facility divided by the energy used to construct, operate and decommission that facility. While EROI is not the only criterion used to make an investment decision, it has been shown that, in technologically advanced societies, energy supplies must exceed a minimum EROI. Furthermore, technological history shows a trend towards higher EROI energy supplies.

EROI calculations have been performed for many components of energy technology: oil wells, wind turbines, photovoltaic modules, biofuels, and nuclear reactors. This report represents the first standalone EROI analysis of nuclear fuel reprocessing (or recycling) facilities.

\section{DEFINITION OF EROI}

Several definitions of EROI exist. For valid comparisons among fuel cycles and for comparison to other types of energy production systems, it is important to state the EROI definition that is being used. R.M. Rotty et. al. did an excellent job of explaining four of these definitions in their 1975 report [3]. In this analysis, two versions of the EROI for nuclear fuel recycling are calculated: Primary EROI and Final EROI.

Primary energy is defined as the heating value of energy taken from the environment at the point where it enters the human-managed energy supply chain. Examples of primary energy carriers include uranium ore, wellhead natural gas and unprocessed biomass.

Primary EROI is the gross sum over the outputs of a process of the primary energy equivalents of those outputs, divided by the gross sum over the inputs of the process of the primary energy equivalents of those inputs. Electrical inputs/outputs are tallied as the heat required to produce them (assuming they could be generated in a thermal power plant fed by a raw natural resource). Fuel inputs/outputs are tallied as the gross inputs to fuel refining processes (generally $100 \%-115 \%$ of the refined product). For the purposes of this analysis, Primary EROI is defined as the ratio of the heating value of all fuels produced to the heating value of all natural resources consumed over the plant life cycle. Primary EROI places all natural resources on an equivalent basis and tallies the resource "tax" that must be paid in order to access more resources.

Final energy is defined as the heating value of energy when it is delivered to the consumer. 
Examples of final energy carriers include electricity, distributed natural gas and purchased gasoline.

Final EROI is the gross sum over the outputs of a process of the final energy delivered by those outputs divided by the gross sum over the inputs of the process of the final energy equivalent of the input carriers. For the purposes of this analysis, Final EROI is defined as the ratio of the electricity produced from recycled fuel to the sum of all energy inputs required over the plant life cycle. Final EROI treats both electricity and fuels on their intrinsic heating value basis.

\section{FUEL CYCLE SCENARIOS}

In this analysis, EROI is calculated for four specific fuel recycle scenarios. Each of these scenarios begins with a resource of used nuclear fuel (UNF) from an initial cycle of low enrichment Uranium oxide (UOX) fuel used in a conventional light water reactor (LWR). This study does not evaluate the EROI for this initial LWR-UOX cycle. Instead, this study considers the used UOX fuel to be a resource that may be recycled in either LWRs or in sodium-cooled fast-spectrum reactors (SFRs) - as either mixed U-Pu oxide (MOX) fuel or as reenriched reprocessed uranium (RepU) in oxide fuel. The four scenarios evaluated are:

- One-pass recycle - Recovered plutonium and uranium are burned as MOX and re-enriched UOX fuel in LWRs.

- Two-pass recycle - After a first pass in LWRs, MOX and UOX fuels are reprocessed for a second pass in LWRs.

- Multi-cycle burner SFR - After a first pass in LWRs, MOX fuel is reprocessed to produce fresh MOX fuel for a burner SFR (conversion ratio $\sim 0.3$ ).

- One-pass breeder SFR - After a first pass in LWRs, MOX fuel is reprocessed to produce fresh MOX fuel for a breeder SFR (conversion ratio 1.2). Excess plutonium from the breeders is burned as MOX in LWRs.

In the SFR scenarios only a limited number of passes through the reactor and recycle system were considered. In the SFR Cycle the potential exists for multiple passes that could produce unrealistically high EROI values.

Many more potential configurations of nuclear fuel cycles could be proposed, ranging from open 'once-through' cycle to partially closed or 'modified open' cycles and to fully closed cycles - each with a wide range of possible technologies and processes. The tools and methodology for EROI analysis described in this report can be used to evaluate those configurations in the future, assuming development of energy intensity data for facilities and processes as needed.

\section{METHODOLOGY}

A bounded Input/Output analysis is used to calculate EROI for nuclear fuel recycling. The inputs are composed of the energy investments required in the construction, use and dismantling of new nuclear fuel facilities. The outputs are the electrical energy delivered by burning the recycled fuel in nuclear reactors.

The system boundaries determine energy inputs and outputs. This analysis includes energy supply/use due to: utilization of reprocessed nuclear fuels in a reactor fleet; petroleum fuel energy use in the construction of reprocessing facilities; energy used in the production of concrete and steel for the construction of reprocessing facilities; electricity use at the reprocessing plant during operation; natural gas use at the reprocessing plant during operation. This analysis, however, does not include energy use due to: transportation of nuclear fuel or depleted uranium to/from recycle center, reactors or repositories; geologic disposal of nuclear fuel; manufacture of cladding; transportation of reagents; transportation of workers. Detailed boundaries were set for each facility.

A spreadsheet tool was constructed by LLNL for the specific purpose of this bounded input/output analysis of nuclear fuel reprocessing facilities. This tool allows the user to enter the parameters of the nuclear fuel cycle and assumptions about energy use in reprocessing. The spreadsheet calculates Primary EROI and Final EROI as defined above. The basic EROI calculation is relatively simple. The complexity is in defining the energy production system to be evaluated, its 'energy boundaries', and in determining reasonable energy content values for the system components. 
TABLE I. Fuel Production and EROI for Four Fuel Cycle Scenarios.

\begin{tabular}{|l|c|c|c|c|}
\hline \multicolumn{1}{|c|}{ Fuel Cycle Scenario } & $\begin{array}{c}\text { Total MOX } \\
\text { (tHM/y) }\end{array}$ & $\begin{array}{c}\text { Total RepU } \\
\text { (tHM/y) }\end{array}$ & $\begin{array}{c}\text { EROI } \\
\text { (Primary } \\
\text { Energy) }\end{array}$ & $\begin{array}{c}\text { EROI } \\
\text { (Final } \\
\text { Energy) }\end{array}$ \\
\hline $\begin{array}{l}\text { One Pass Recycle } \\
\text { UNF } \rightarrow \text { Recycle } \rightarrow \text { LWR } \rightarrow \text { Disposal }\end{array}$ & 100.0 & 58.5 & 108.2 & 71.6 \\
\hline $\begin{array}{l}\text { Two Pass Recycle } \\
\text { UNF } \rightarrow \text { Recycle } \rightarrow \text { LWR } \rightarrow \text { Recycle } \rightarrow \text { LWR } \rightarrow \\
\text { Disposal }\end{array}$ & 140.1 & 52.0 & 122.0 & 81.0 \\
\hline $\begin{array}{l}\mathbf{2}^{\text {nd }} \text { Pass Recycle to Multi-pass SFR Burners } \\
\text { UNF } \rightarrow \text { Recycle } \rightarrow \text { LWR } \rightarrow \text { Recycle } \rightarrow \text { SFR Burner } \\
(\text { Pu) and LWR (RepU) } \rightarrow \text { Disposal }\end{array}$ & 138.9 & 48.4 & 134.8 & 96.0 \\
\hline $\begin{array}{l}\mathbf{2}^{\text {nd }} \text { Pass Recycle to Single-pass SFR Breeders } \\
\text { UNF } \rightarrow \text { Recycle } \rightarrow \text { LWR } \rightarrow \text { Recycle } \rightarrow \text { SFR Breeder } \\
\rightarrow \text { Recycle } \rightarrow \text { LWR } \rightarrow \text { Disposal }\end{array}$ & 131.2 & 71.3 & 138.9 & 98.3 \\
\hline
\end{tabular}

\section{RESULTS}

Table I summarizes the major findings of this analysis. All systems assume the use of an $800 \mathrm{tHM} / \mathrm{yr}$ recycling facility and appropriately scaled MOX fabrication, UOX fabrication, RepU processing and enrichment facilities. Fuel cycles were considered to be at steady-state equilibrium for this analysis.

\section{CONCLUSIONS}

Under a wide range of assumptions, the construction, operation and decommissioning of a used fuel recycling facility and reuse of the fuel resource provides a high EROI. According to industrial data from AREVA (and conservative estimates where industrial data could not be found), the EROI for the nuclear fuel recycling enterprise should range from 72 to 98 on a final energy basis, depending on the fuel cycle employed. This conclusion depends on the ability to leverage the current LWR fleet to burn recycled fuel, and does not take into account the energy required to dispose of the waste from recycling.

The "energy return" part of the EROI calculation is solely a function of the fuel cycle chosen. Cycles that produce greater quantities of useable fuel from similarly sized facilities, and cycles that burn fuel in higher efficiency reactors are favored in EROI calculations. Technological readiness (of fast reactors and high burnup fuels) is a key component of the nuclear industry's ability to realize these advantages.

The "energy invested" part of the EROI calculation illuminates the challenges to efficiently recycling nuclear fuel. Operation of the head-end, comprising $40 \%$ - $50 \%$ of the total life cycle energy budget, was previously estimated to be $10 x$ lower than it is today (See Ref. 1, Section A.1.2.1). Advances in centrifuge technology have surpassed that era's prediction of energy efficiency improvements, so it is not unreasonable to assume that some improvement in head-end energy efficiency is possible. Potential large decommissioning energy use represents a 'lesson learned', and an area with important residual uncertainty. Estimated decommissioning energy for the recycling facility alone, comprising nearly another one-third of the total life cycle energy investment into the recycling enterprise, might be reduced if the need for West Valley Demonstration Project-like environmental remediation can be avoided. Such reductions in the denominator would noticeably increase EROI.

However, EROI is not the sole criterion (or even a primary criterion) on which an investment decision should be made, so these conclusions must be weighed against the financial costs and benefits of recycling as well as the environmental and proliferation risks and benefits associated with recycling.

\section{FUTURE APPLICABILITY}

The EROI metric can be used to evaluate more fuel cycles than the four that have been analyzed here. Specifically, EROI may be used to compare recycling options with fuel cycles that do not employ recycling. That comparison will relax the assumption that this analysis did not take credit for offsetting energy inputs into the production of LWR fuel from virgin uranium. 
Another assumption made in this analysis was that all fuel cycles operated at steady-state. For twoor multi-pass fuel cycles, material flows would resemble a one-pass cycle for the first several years of operation, and then would evolve towards steady state as more used/recycled fuel becomes available for processing. A more precise EROI could be calculated with these startup effects (and the attendant requirement for short-term fuel storage) taken into account.

The principal challenge to further application of this methodology is development of a logical system description and analysis boundary, and obtaining defensible energy content values. This initial analysis can provide a template for such future studies.

\section{REFERENCES}

1. A. J. SIMON, MAX FRATONI, CLARA

SMITH, AND WILLIAM HALSEY, "Energy Return on Investment - Fuel Recycle”, FCR\&D-SYSA2011-000317, LLNL-TR-497511, Lawrence

Livermore National Laboratory, (2011).

2. PAUL MURRAY, et. al.,," "Energy Return on Investment from the Operation of Recycle Facilities", Contract No. DOE-NE0000291, RPT-3005597-000, Areva Federal Services LLC, (2011).

3. R. M. ROTTY, A. M. PERRY, AND D. B. REISTER, "Net Energy from Nuclear Power", IEA75-3, Institute for Energy Analysis, Oak Ridge Associated Universities, (1975). 